\title{
Identification of Candida Species from Clinical Isolates and Their Antifungal Susceptibility Pattern
}

\author{
Deepthi K. N. ${ }^{1}$, Ardra R. Menon², Prithi Nair K. ${ }^{3}$ \\ ${ }^{1}$ Department of Microbiology, Government Medical College, Thrissur, Kerala, India. \\ ${ }^{2}$ Department of Microbiology, Government Medical College, Thrissur, Kerala, India. \\ ${ }^{3}$ Department of Microbiology, Government Medical College, Thrissur, Kerala, India.
}

\section{ABSTRACT}

\section{BACKGROUND}

With increasing use of antibacterial and cytotoxic drugs, lethal invasive Candidiasis is on the rise, with almost half of the cases being caused by non albicans Candida species (NAC). Frequent use of azoles for empirical therapy has also led to their increased resistance. We wanted to characterise Candida species isolated from various clinical specimens and assess their susceptibility pattern to Fluconazole and Voriconazole.

\section{METHODS}

A total of 100 consecutive Candida species isolated from various clinical specimens in our institute from January 2016 to December 2016 were included in the study. Standard yeast identification protocol and CHROM agar were used for speciation and their antifungal susceptibility pattern was found by disc diffusion method.

\section{RESULTS}

Out of the 100 isolates, $C$. tropicalis was the predominant isolate (47\%), followed by C. albicans (31\%), C. parapsilosis (16\%) and C. krusei (6\%). Females (57\%) were more affected and maximum number of patients was above 60 years (24\%). Diabetes mellitus (21\%) was the major predisposing factor for Candida, followed by broad spectrum antibiotic therapy (14\%). Isolates were more susceptible to Voriconazole (99\%) than Fluconazole (87\%). NAC spp. showed more resistance to Fluconazole (17.4\%) than C. albicans (3.3\%). Only one isolate of C. krusei $(16.6 \%)$ showed resistance to Voriconazole.

\section{CONCLUSIONS}

Due to the increasing incidence of azole resistant NAC spp., the species level identification of Candida species, along with their anti-fungal susceptibility patterns can help the clinicians in formulating a treatment protocol and can help in decreasing the mortality and morbidity.

\section{KEY WORDS}

Candida albicans, Non-albicans Candida, CHROMagar, Antifungal Susceptibility Testing, Fluconazole, Voriconazole
Corresponding Author: Dr. Ardra R. Menon, Anugraha, Gandhinagar, Mannuthy PO. Thrissur-680651, Kerala, India.

E-mail:drardra@yahoo.co.in

DOI: $10.14260 /$ jemds/2020/396

Financial or Other Competing Interests: None.

How to Cite This Article: Deepthi KN, Menon AR, Nair PK. Identification of candida species from clinical isolates and their antifungal susceptibility pattern. J. Evolution Med. Dent. Sci. 2020;9(24):1813-1817, DOI: 10.14260/jemds/2020/396

Submission 15-02-2020, Peer Review 19-05-2020 Acceptance 26-05-2020, Published 15-06-2020.

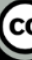




\section{BACKGROUND}

Candida, a genus of yeast are ubiquitous organisms found on many plants and are a part of normal flora of the alimentary tract of mammals and the mucocutaneous membranes of humans. Candida causes a diverse range of opportunistic human infections from mild superficial to the life-threatening invasive ones. Adherence to host tissues, medical devices, biofilm formation, and secretion of extracellular hydrolytic enzymes has facilitated the switch of Candida spp. from commensal to a potent pathogen. ${ }^{1}$ The increasing incidence of HIV infections, use of steroids and broad spectrum antibiotics, organ transplantations, advanced life supports and prosthetic devices, all have led to the increasing incidence of Candida infections. Candida albicans is generally considered as the most common and the pathogenic member of the genus. ${ }^{2}$

More recently, there has been a significant shift towards non-albicans Candida (NAC) infections, which have shown variable resistance to azoles. In developed countries, 40-60\% of isolates are C albicans; whereas in India there is a preponderance of NAC spp. ${ }^{3}$ NAC is a heterogeneous group, of which 19 species are implicated in human infections. $C$. tropicalis, C. glabrata, C. krusei and C. parapsilosis are the most commonly reported NAC spp. The NAC spp. may represent selection of less susceptible species like $\mathrm{C}$ glabrata and $\mathrm{C}$ krusei which is intrinsically resistant to Fluconazole. Even though $C$. tropicalis is generally considered as a Fluconazole-susceptible species, recent studies have shown emergence of Fluconazole resistance. ${ }^{4}$

Invasive Candida infections are one of major causes of morbidity and mortality in immunocompromised patients. Therefore, in patients with risk factors for Candidemia, clinicians empirically treat the infection with antifungal agents, most commonly azoles. To avoid selection of less susceptible NAC spp. by empirical azole treatment or prophylaxis, identification of the infecting species of Candida isolate is essential for initiation of early and effective therapy. Although there are rapid and reliable commercial systems and molecular diagnostic methods available for species identification of Candida isolates, in a resource poor country like ours conventional techniques remain the mainstay in most clinical microbiology laboratories. ${ }^{4}$ In such setting, usage of CHROMagar which contains chromogenic (hexosaminidase) substrates that react with species-specific enzymes secreted by yeast cells, for species identification would be of benefit for easy and rapid speciation. ${ }^{3}$

As the prevalence of NACs spp. significantly vary according to countries and health care facilities, species identification also play a part in formulating local therapeutic guidelines. ${ }^{4}$ So the present study was undertaken to characterise the Candida isolates in our geographic area and to determine the antifungal susceptibility pattern of the isolates to Fluconazole and Voriconazole so as to formulate an empirical treatment policy.

\section{METHODS}

A descriptive study was undertaken in the department of Microbiology, Govt. Medical College, Thrissur, Kerala for a period of 1 year from January 2016 to December 2016. All pure isolates of Candida spp. from various clinical specimen during the period of study were included in the study. (based on the previous 3 years hospital records, a sample size of 80 100 was calculated). A total of 100 consecutive and nonrepetitive pure cultures of Candida from various clinical specimens like blood, urine, sputum, swabs, nail clippings, endotracheal tube tip, skin scrapings, exudates etc. received in the Diagnostic Microbiology section of central laboratory were studied. All the yeast isolates (white to cream-coloured, pasty and smooth colonies) grown on Blood agar were confirmed by Gram staining which showed Gram positive budding cells with or without pseudohyphae. Single colony from the Blood agar was inoculated on Sabouraud dextrose agar and incubated at $37^{\circ} \mathrm{C}$ for 24 hours. Germ tube test was done for all the isolates and the positives were identified as either $C$. albicans or $C$. dubliniensis. Species identification was done by using Corn meal agar, Sabouraud's Dextrose broth, Christensen's urea agar, Sugar assimilation and sugar fermentation. The isolates were further processed for Candida speciation on CHROMagar (HiMedia, Mumbai, India). After incubation at $37{ }^{\circ} \mathrm{C}$ for $24-48 \mathrm{hrs}$, Candida species were identified by type and colour of the colonies as per manufacturer's instructions. Type of the growth and colour of isolates on CHROMagar Candida plates; C. albicans light green, C. parapsilosis off white to pink, Candida krusei purple, fuzzy and $C$. tropicalis blue to purple.

Antifungal susceptibility testing by disc diffusion method to Fluconazole and Voriconazole were studied for all the isolates of Candida as recommended by Clinical and Laboratory Standards Institute (CLSI) M44-A2 document guidelines using Fluconazole disc $(25 \mu \mathrm{g})$ and Voriconazole disc $(1 \mu \mathrm{g})$ (HiMedia, Mumbai, India). ${ }^{5}$

\section{Statistical Analysis}

Data was entered in Microsoft Excel. Qualitative data was described using proportion and percentages.

\section{RESULTS}

A total of 100 Candida spp. was isolated from various clinical samples (table 1). Majority of the isolates were from Urine (46\%), blood culture (13\%) and sputum (12\%). Gender-wise distribution showed that $57 \%$ Candida isolates were from females and $43 \%$ from males. Maximum number of patients were above 60 years (24\%) followed by reproductive age group (21\%) and newborn (21\%). Out of the 100 isolates of Candida, Non-albicans Candida species predominated with 69 $\%$ and Candida albicans $31 \%$. Among the NAC spp., $C$. tropicalis (47\%) was the most common isolate followed by $C$. parapsilosis (16\%) and C. krusei (6\%). C. albicans was the predominant isolate from blood, high vaginal swab, exudates and pus swab. C. tropicalis was the predominant isolate from urine and sputum. Out of the 100 isolates, 67 were high risk groups, of which diabetes mellitus constituted $21 \%$, followed by prolonged antibiotic therapy (table 2).

Among the total 100 Candida isolates, $87 \%$ were sensitive to Fluconazole and $99 \%$ to Voriconazole. In this study, only $3.3 \%$ of $C$. albicans was found to be resistant to Fluconazole. 
Among the NAC spp., C. tropicalis and. C parapsilosis showed $8.5 \%$ and $12.5 \%$ Fluconazole resistance respectively whereas $100 \%$ of C krusei were resistant to Fluconazole. All the isolates of $C$. albicans, C. tropicalis and C. parapsilosis were sensitive to Voriconazole. One isolate of C. krusei (16.6\%) showed resistance to Voriconazole (table 3).

\begin{tabular}{|cccccc|}
\hline Specimens & $\begin{array}{c}\text { Candida } \\
\text { albicans }\end{array}$ & $\begin{array}{c}\text { Candida } \\
\text { tropicalis }\end{array}$ & $\begin{array}{c}\text { Candida } \\
\text { parapsilosis }\end{array}$ & $\begin{array}{c}\text { Candida } \\
\text { krusei }\end{array}$ & Total \\
Urine & 8 & 31 & 7 & 0 & 46 \\
Blood & 5 & 4 & 1 & 3 & 13 \\
Sputum & 4 & 6 & 2 & 0 & 12 \\
Exudates & 3 & 2 & 2 & 2 & 9 \\
High vaginal & 6 & 0 & 2 & 0 & 8 \\
swab & 2 & 1 & 1 & 1 & 5 \\
Pus swab & 2 & 1 & 1 & 0 & 3 \\
Skin scraping & 1 & 1 & 0 & 0 & 2 \\
Nail clipping & 1 & 1 & 0 & 0 & 2 \\
Catheter tip & 1 & $\mathbf{4 7}$ & $\mathbf{1 6}$ & $\mathbf{6}$ & $\mathbf{1 0 0}$ \\
Total & $\mathbf{3 1}(\mathbf{3 1}$ ) & $\mathbf{4 7}$ &
\end{tabular}

Table 1. Species Distribution of Candida Isolates in Various Specimens

\begin{tabular}{|cccccc|}
\hline $\begin{array}{c}\text { Predisposing } \\
\text { Factors }\end{array}$ & $\begin{array}{c}\text { Candida } \\
\text { albicans }\end{array}$ & $\begin{array}{c}\text { Candida } \\
\text { tropicalis }\end{array}$ & $\begin{array}{c}\text { Candida } \\
\text { parapsilosis }\end{array}$ & $\begin{array}{c}\text { Candida } \\
\text { krusei }\end{array}$ & Total \\
Diabetes mellitus & 12 & 7 & 2 & 0 & 21 \\
Antibiotic therapy & 6 & 5 & 3 & 0 & 14 \\
Pregnancy & 4 & 4 & 1 & 0 & 9 \\
Sepsis & 3 & 3 & 1 & 2 & 9 \\
Devices in situ & 3 & 3 & 1 & 0 & 7 \\
Malignancy & 1 & 2 & 0 & 1 & 4 \\
Immuno & 1 & 1 & 0 & 1 & 3 \\
suppression & $\mathbf{3 0}$ & $\mathbf{2 5}$ & $\mathbf{8}$ & $\mathbf{4}$ & $\mathbf{6 7}$ \\
Total & Table 2. Species Distribution According to Predisposing Factors \\
\hline \multicolumn{6}{|r}{} \\
\hline
\end{tabular}

\begin{tabular}{|ccccc|}
\hline Antifungal & $\begin{array}{c}\text { Candida } \\
\text { albicans } \\
(\mathbf{n = 3 1 )}\end{array}$ & $\begin{array}{c}\text { Candida } \\
\text { tropicalis } \\
(\mathrm{n}=47)\end{array}$ & $\begin{array}{c}\text { Candida } \\
\text { parapsilosis } \\
(\mathbf{n}=16)\end{array}$ & $\begin{array}{c}\text { Candida } \\
\text { krusei } \\
(\mathbf{n}=6)\end{array}$ \\
$\begin{array}{c}\text { Fluconazole } \\
\text { sensitive } \\
\begin{array}{c}\text { Voriconazole } \\
\text { sensitive }\end{array}\end{array}$ & $30(96.7 \%)$ & $43(91.5 \%)$ & $14(87.5 \%)$ & 0 \\
\hline \multicolumn{4}{|c|}{ Table 3. Azole Susceptibility Pattern of Candida Species } \\
\hline
\end{tabular}

\section{DISCUSSION}

Out of the 100 isolates obtained from various clinical specimens, the majority of Candida species were from urine (46\%), blood (13\%) and sputum (12\%). Of these $>80 \%$ of urinary Candida isolates belonged to NAC spp. Our observation is similar to that of Arasi Samyuktha $\mathrm{A}^{\text {et }} \mathrm{al}^{3}$ and Khadka $\mathrm{S}$ et $\mathrm{al}^{1}{ }^{1}$ where more than half of the urinary Candida isolates belonged to NAC spp. In the most recent study conducted by Pfaller et al for the SENTRY Antimicrobial Surveillance Programme, Candida species was found out to be the seventh most common nosocomial pathogen and caused $25 \%$ of all urinary tract infections. ${ }^{6}$

In the present study, the isolation rate of Candida spp. was highest in the age group more than 60 years (24\%) followed by reproductive age group $(21 \%)$ and newborn $(21 \%)$. This emphasises the fact that Candida spp. is the most important cause of opportunistic infections worldwide in extremes of age as shown in other studies of Arasi Samyuktha $\mathrm{A} \mathrm{et} \mathrm{al}^{3}$ and Dharwad $\mathrm{S}$ et al $^{7}$ Candida isolates were found to be highest in female $(57 \%)$ with a female to male ratio of $1.3: 1$. Although in majority of studies, male preponderance is noted, female predominance was noted in study by Dharwad S et al ${ }^{7}$ with female to male ratio 1.7:1 respectively. The reason for this disparity may be due to the higher number of female samples selected for the study.
CHROMagar offered a rapid and reliable method for identification of clinically important Candida species when compared with the traditional techniques. Out of the 100 isolates of Candida, Non-albicans Candida species predominated with $69 \%$ isolation. Among the NAC spp., $C$. tropicalis $(47 \%)$ was the most common isolate irrespective of the nature of the specimen, followed by $C$. parapsilosis (16\%) and $C$. krusei $(6 \%)$. This result is consistent with various studies conducted worldwide including India where NAC spp. Outnumbered $C$. albicans with incidence ranging from 54$74 \%$ which suggest the emergence of NAC spp. as important pathogens. ${ }^{3,7-11}$ The possible reason for this may be the indiscriminate use of antifungals which eliminates more sensitive $C$. albicans and selects azole resistant NAC spp. However, higher incidence of $C$. albicans has been seen in numerous studies.1,12-14 Among the Non-albicans Candida, various studies have reported $C$. tropicalis as the most predominant species in India.3,7.10.11,13 Variation in predominance of Candida species observed in different studies may be due to the change in the environmental conditions, diversity in the study population, or the institutional based protocol for the usage of antifungal agents. In our study, $C$. albicans was the predominant isolate from blood, high vaginal swab, exudates and pus swab. C. tropicalis was the predominant isolate from urine. C. glabrata has emerged as an important opportunistic pathogen worldwide with concern over the increase in azole resistance. But in the present study, there was no isolate of the species.

Candidiasis occurs mostly in the presence of risk factors which promotes its overgrowth. In this study, Type II diabetes mellitus was the leading risk factor (21\%) along with antibiotics, immunosuppressants, malignancy, pregnancy and catheters. A study by Dharwad S et al also showed Diabetes as a frequent risk factor with incidence of $32 \% .^{7}$ An increase of glucose concentration can promote the growth of Candida; Diabetes mellitus decreases the chemotactic factors and impairs phagocytosis. C. albicans was the predominant species in all the predisposing factors. Broad spectrum antibiotic usage was the second most frequently associated risk factor (14\%). The most important effect of antibiotics is the elimination and alteration of the normal bacterial flora. It is an established fact that high hormone level leads to a proportional increase in the glycogen content of the vagina increasing prevalence of genital Candidiasis in pregnancy. In the present study, 9 pregnant women had infection.

Sepsis (9\%) and urinary and indwelling catheters (7\%) were the other significant risk factors, which can cause an increased risk of blood stream infections by Candida. In our study, the second most common specimen was blood (13\%). In studies by Adhikary et al and Dharwad $S$ et al, the rate of Candidemia was $19 \%$ and $16 \%$ respectively.7,10 Dismissal of the isolation of the Candida spp. from a single blood culture as a skin contaminant could lead to a delay in administering potentially lifesaving therapy. It is said to consider all blood cultures that yield Candida spp. as significant until proven otherwise. ${ }^{7}$

Azoles are safe and effective agents for the treatment of Candidiasis and have gradually replaced Amphotericin B. However, resistance to azoles is now on the rise. According to the SENTRY Program findings, aside from intrinsically 
Fluconazole-resistant species, such as C. krusei and C.auris, increasing rates of acquired resistance to Fluconazole have been noted in other NAC spp. including C.glabrata, C. parapsilosis, and C.tropicalis in India. Voriconazole has been demonstrated to have useful clinical activity in treating mucosal and invasive forms of Candidiasis. The clinical indication for using Voriconazole in invasive Candidiasis has been for oral step-down therapy in patients with $C$. krusei and Fluconazole-resistant, Voriconazole-susceptible C. glabrata. ${ }^{6}$

Among the total 100 Candida isolates in the present study, $13 \%$ of the total isolates were resistant to Fluconazole and only $1 \%$ to Voriconazole. The rate of Fluconazole resistance was highest in C. parapsilosis (12.5\%) and $C$. tropicalis (8.5\%) with only $3.2 \%$ of $C$. albicans showing Fluconazole resistance. All the 6 isolates of C. krusei (100\%), which are considered to be intrinsically resistant to Fluconazole, were resistant to it. Various studies from the Indian subcontinent shows Fluconazole resistance ranging from 0 to $37 \%$ for $C$. albicans.1,7,9,11,15 In studies by Adhikary et al, Khadka S et al and Dharward S et al, 19\%, $20 \%$ and $25 \%$ of Fluconazole resistance was noted for $C$. tropicalis respectively. ${ }^{1.7 .10} \mathrm{C}$. parapsilosis has become more significant and prevalent over the past two decades and is now one of the leading causes of invasive Candidiasis. ${ }^{6}$ In a multi-centre laboratory based surveillance study conducted in India on Clonal Fluconazole resistant $C$. parapsilosis, over a period of 3 years from $2015-17,32 \%$ were found to be non-susceptible to Fluconazole. ${ }^{16}$ These were considered as hospital strains as indiscriminate use of Fluconazole for fungal infections have led to the emergence and subsequent nosocomial transmission of Fluconazole-resistant strains.

All the isolates of $C$. albicans, $C$. tropicalis and $C$. parapsilosis were sensitive to Voriconazole. One isolate of $C$. krusei $(16.6 \%)$ showed resistance to Voriconazole. In most of the Indian studies, the C. albicans was $100 \%$ sensitive to Voriconazole.7,10,11,17 But in a study by Dharward $\mathrm{S}$ et al $C$. tropicalis showed $12.5 \%$ and C. krusei showed $20 \%$ Voriconazole resistance and in another study by Kaur R et al C. tropicalis and C parapsilosis showed $70 \%$ and $30 \%$ Voriconazole resistance respectively.7,15 In our study, Candida isolates displaying resistance to Fluconazole were susceptible to Voriconazole which beyond doubt proves it to be an effective in the treatment of Candidemia caused by Fluconazole resistant strains.

\section{CONCLUSIONS}

The shift in epidemiology from $C$. albicans to NAC spp. with increased azole resistance emphasizes the need for constant monitoring of Candida species distribution and continuously monitoring antifungal susceptibility patterns. Use of azoles especially Fluconazole should be controlled by good clinicalmicrobiological correlation to prevent resistance by selective pressure. This will help in the optimum management of the patients, helping in decreasing the overall morbidity and mortality.

\section{ACKNOWLEDGEMENT}

We express our sincere thanks to all the teaching and nonteaching staff in the Department of Microbiology, Government Medical College, Thrissur, for their constant support and co-operation.

\section{REFERENCES}

[1] Khadka S, Sherchand JB, Pokhrel BM, et al. Isolation, speciation and antifungal susceptibility testing of Candida isolates from various clinical specimens at a tertiary care hospital, Nepal. BMC Res Notes 2017;10(1):218.

[2] Sasikala G, Udaysri B. Speciation and antifungal susceptibility profiles of Candida isolates from vaginitis patients attending STD clinic at a tertiary care hospital. J NTR Univ Health Sci 2018;7(2):94-7.

[3] Samyuktha AA, Saikuma C. Isolation, identification and speciation of candida species from various clinical specimens in a tertiary care hospital in Chennai. Sch J App Med Sci 2017;5(8F):3460-8.

[4] Deorukhkar SC, Roushani S. Identification of Candida species: conventional methods in the era of molecular diagnosis. Ann Microbiol Immunol 2018;1(1):1002.

[5] CLSI. Method for antifungal disk diffusion susceptibility testing of yeasts; approved Guideline Second Edition. CLSI Document M44-A2. Wayne, PA: Clinical and Laboratory Standards Institute 2009.

[6] Pfaller MA, Diekema DJ, Turnidge JD, et al. Twenty years of the SENTRY antifungal surveillance program: results for Candida species from 1997-2016. Open Forum Infectious Diseases 2019;6(1):S79-94.

[7] Dharward S, Dominic RM. Species identification of Candida isolate in various clinical specimens with their antifungal susceptibility patterns. JCDR 2011;5(6):117781.

[8] Falagas ME, Roussos N, Vardakas KZ. Relative frequency of albicans and the various non-albicans candida spp. among candidemia isolates from inpatients in various parts of the world: a systematic review. Int J Infect Dis 2010;14(11):e954-66.

[9] Vijaya D, Harsha TR, Nagaratnamma T. Candida speciation using CHROM agar. J Clin Diagn Res 2011;5(4):755-7.

[10] Adhikary R, Joshi S. Species distribution and anti-fungal susceptibility of Candidaemia at a multi super-speciality center in Southern India. Indian J Med Microbiol 2011;29(3):309-11.

[11] Mokaddas EM, Al-Sweih NA, Khan ZU. Species distribution and antifungal susceptibility of Candida bloodstream isolates in Kuwait: a 10 year study. J Med Microbiol 2007;56(Pt 2):255-9.

[12] Kanna BV, Kumar GA, Swapna M, et al. Isolation and identification of candida species from various clinical samples in a tertiary care hospital. Int J Res Med Sci 2017;5(8):3520-2.

[13] Jindal N, Gill P, Aggarwal A. An epidemiological study of vulvovaginal candidiasis in women of childbearing age. Indian J Med Microbiol 2007;25(2):175-6. 
[14] Manjunath V, Vidya GS, Sharma A, et al. Speciation of Candida by Hicrome agar and sugar assimilation test in both HIV infected and non-infected patients. Int J Biol Med Res 2012;3(2):1778-82.

[15] Kaur R, Jaggi S, Dhakad MS, et al. An etiological and antifungal profile of candidemia in children. Int J Community Med Public Health 2019;6(9):3899-904.

[16] Singh A, Singh PK, de Groot T, et al. Emergence of clonal fluconazole-resistant Candida parapsilosis clinical isolates in a multicentre laboratory-based surveillance study in India. J Antimicrob Chemother 2019;74(5):1260-8.

[17] Lavanya V, Pavani P, Kailasanatha Reddy B. Speciation and antifungal susceptibility pattern of Candida isolates from vulvovaginitis patients attending a tertiary care hospital in South India. IAIM 2019;6(2):62-8. 